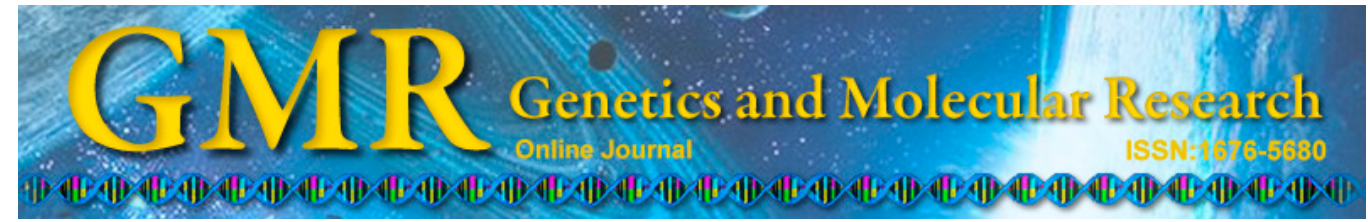

\title{
Effects of changes in serum endostatin and fibroblast growth factor 19 on the chemotherapeutic sensitivity in acute myeloid leukemia patients
}

\author{
Y.Z. Su${ }^{1}$, C.B. Wang' ${ }^{2}$ Y. Zhou' ${ }^{2}$ and N.T. Sun ${ }^{2}$ \\ ${ }^{1}$ Department of Hematology, \\ The First Affiliated Hospital of Shantou University Medical College, \\ Shantou, China \\ ${ }^{2}$ Department of Tumor and Hematology, \\ Yancheng Hospital Affiliated to Southeast University \\ (The Third People's Hospital of Yancheng), Yancheng, China \\ Corresponding author: C.B. Wang \\ E-mail: chunbinwang@126.com
}

Genet. Mol. Res. 14 (2): 5181-5187 (2015)

Received September 30, 2014

Accepted December 11, 2014

Published May 18, 2015

DOI http://dx.doi.org/10.4238/2015.May.18.8

\begin{abstract}
The present study aimed to explore the changes in serum endostatin and fibroblast growth factor 19 (FGF-19) in acute myeloid leukemia patients, and to determine their effects on chemotherapeutic sensitivity. Sixty acute myeloid leukemia patients and 30 healthy controls were included in the study. Patient serum endostatin and FGF-19 levels were measured on admission, and then, standard chemotherapy was administered. The patients were divided into 2 groups according to chemotherapeutic effects: 21 patients in the chemotherapeutic sensitivity group (complete remission + partial remission) and 39 in the chemotherapeutic resistance group (no remission + degradation). A receiver operating characteristic (ROC) curve was used to analyze the relationship of serum endostatin and
\end{abstract}


FGF-19 levels with chemotherapeutic sensitivity in acute myeloid leukemia patients. The levels of serum endostatin and FGF-19 in acute myeloid leukemia patients before chemotherapy were significantly higher than those in the control group. Moreover, these levels significantly decreased after chemotherapy $(\mathrm{P}<0.01)$. The levels of serum endostatin and FGF-19 in the chemotherapeutic sensitivity group were lower than those in the chemotherapeutic resistance group, both before and after chemotherapy ( $\mathrm{P}<0.05$ and $\mathrm{P}<0.01$, respectively). ROC curve analysis showed that the predictive values of endostatin and FGF-19 were good, and there was no significant difference between these results. In conclusion, serum endostatin and FGF-19 can be used as predictors of chemotherapeutic sensitivity for acute myeloid leukemia patients, and may be important for determining prognosis.

Key words: Acute myeloid leukemia; Fibroblast growth factor 19; Endostatin; Chemotherapeutic sensitivity

\section{INTRODUCTION}

Acute myeloid leukemia is a common malignant disease of the blood. It has a high incidence and accounts for approximately $60 \%$ of all leukemia; therefore, it is a serious threat to human health (Won et al., 2013; Bernal et al., 2014). Patients with acute myeloid leukemia often have a variety of diseases. Because of cytogenetic and other factors, complete remission after chemotherapy is low, patient prognosis is poor, the disease-free survival period is short, and the 5-year survival rate is only approximately $10 \%$ (Lin et al., 2013; Oberoi et al., 2014). Thus, early intervention is important for the treatment and prognosis of patients with acute myeloid leukemia. Serum endostatin is a potent endogenous vascular growth inhibitor; it can effectively inhibit the growth and transition of endothelial cells, and reduce primary tumor growth and the metastasis rate. No drug resistance or toxic side effects have been observed after its use. The level of endostatin has been correlated with the progression and prognosis of a variety of malignant tumors (Liu et al., 2012; Xia et al., 2012; Moiseev et al., 2013). Fibroblast growth factor 19 (FGF-19) belongs to the FGF family, and is often expressed in the terminal ileum, brain, skin, and other sites. It can be involved in the metabolism of bile acid, glucose, vitamin D, and can affect the transformation and prognosis of diseases to a certain extent (Rotschafer et al., 2013; Gacche and Meshram, 2014). To date, few studies have examined variations in serum endostatin and FGF-19 in patients with acute myeloid leukemia and those with chemotherapeutic sensitivity, and its effect on prognosis. This study focused on changes in serum endostatin and FGF-19 in patients with acute myeloid leukemia before and after chemotherapy, and analyzed the relationship with chemotherapeutic sensitivity.

\section{MATERIAL AND METHODS}

\section{General data}

A total of 60 patients with acute myeloid leukemia admitted to our hospital and 30 healthy controls were included in this study. All patients did not undergo operative chemo- 
therapy or targeted therapy, in accordance with the World Health Organization standards. Moreover, none of the patients had non-acute panmyelosis disease, bone marrow fibrosis, or myeloma. Of the patients with leukemia, 28 were men with an age range of 21-82 years, and a median age of $55.63 \pm 24.18$ years; 32 were women, with an age range of 23-80 years old, and a median age of $55.14 \pm 25.61$ years. Of the healthy controls, 14 were men with an age range of 22-73 years, and a median age of $53.24 \pm 19.68$ years; 16 were women with an age range of 20-81 years, and a median age of $56.42 \pm 24.90$ years. There were no significant differences in gender, age, or other general data between the two groups $(\mathrm{P}>0.05)$. This study was conducted in accordance with the declaration of Helsinki and with the approval of the ethics committee of Yancheng Hospital affiliated to Southeast University. Written informed consent was obtained from all participants.

\section{Detection methods}

Serum endostatin and FGF-19 levels were analyzed in 30 controls and 60 leukemia patients after admission over the same time. The leukemia patients received neoadjuvant chemotherapy, and their serum endostatin and FGF-19 levels were reviewed after 2 weeks. On the second morning, $5 \mathrm{~mL}$ fasting venous blood was drawn from the elbow of all the subjects. After 2-week chemotherapy, $5 \mathrm{~mL}$ fasting venous blood was drawn from the elbow of leukemia patients, placed at room temperature for approximately $30 \mathrm{~min}$, and centrifuged at 5000 revolutions/min for $10 \mathrm{~min}$. The serum was separated and placed at a constant temperature of $-20^{\circ} \mathrm{C}$ for preservation and future measurement. The detection of endostatin and FGF-19 levels were determined using kits and by following manufacturer protocols. The BIO-RAD Model 550 (Hercules, CA, USA) was also used. Endostatin levels were quantified using a human endostatin sandwich enzyme immunoassay kit (Chemicon International Inc., Temecula, CA, USA). FGF-19 levels were analyzed using a FGF-19 Quantikine ${ }^{\circledR}$ enzyme-linked immunosorbent assay kit (R\&D Systems Inc., Minneapolis, MN, USA).

\section{Treatment methods}

All the leukemia patients received standard chemotherapy for at least 2 weeks by using the HA induction chemotherapy scheme (homoharringtonine, $2 \mathrm{mg} / \mathrm{day}$, and cytarabine, $50 \mathrm{mg} /$ day, for a total of 14 days) or the IA induction chemotherapy scheme (idarubicin, 6-8 $\mathrm{mg} / \mathrm{m}^{2} \times 3$ days and cytarabine, $100 \mathrm{mg} /$ day $\times 7$ days). Consolidation chemotherapy consisted of the DA scheme (daunorubicin, $40 \mathrm{mg} / \mathrm{m}^{2}$, and cytarabine, $100 \mathrm{mg} /$ day), HA scheme, or IA scheme. After chemotherapy, the curative effects were evaluated, and the patients' symptoms were observed.

\section{Curative effect evaluation}

Patients were divided into those who achieved complete remission (CR), partial remission (PR), no remission (SD), or degradation (PD) on the basis of their clinical profile and peripheral blood and bone marrow analysis, according to the third version of "Standard of diagnosis and curative effect for leukemia". CR + PR represented chemotherapeutic sensitivity and $\mathrm{SD}+\mathrm{PD}$, chemotherapeutic resistance. 


\section{Statistical analysis}

All data were analyzed using the SPSS 15.0 software (SPSS Inc., Chicago, IL, USA). The measurement data were compared using the $t$-test, and receiver operating characteristic (ROC) curves were drawn. The relationships between the serum endostatin and FGF-19 levels and the chemotherapeutic sensitivity were analyzed. P values $<0.05$ were considered to be statistically significant.

\section{RESULTS}

\section{Changes in serum endostatin and FGF-19 levels in leukemia patients before and after chemotherapy}

The levels of serum endostatin and FGF-19 in leukemia patients were higher than those in the control group, and these levels significantly decreased after chemotherapy $(\mathrm{P}<$ 0.01 ; Table 1).

Table 1. Changes of serum endostatin and FGF-19 in patients with leukemia before and after chemotherapy.

\begin{tabular}{|c|c|c|c|c|c|}
\hline \multirow[t]{2}{*}{ Groups } & \multirow[t]{2}{*}{ Cases } & \multicolumn{2}{|c|}{ Endostatin $(\mu \mathrm{g} / \mathrm{L})$} & \multicolumn{2}{|c|}{ FGF-19 (ng/L) } \\
\hline & & Before chemotherapy & After chemotherapy & Before chemotherapy & After chemotherapy \\
\hline Patients with leukemia & 60 & $28.68 \pm 15.26$ & $18.14 \pm 6.21$ & $250.66 \pm 45.81$ & $140.24 \pm 26.37$ \\
\hline Control group & 30 & $13.42 \pm 5.74$ & $13.42 \pm 5.74$ & $101.58 \pm 34.65$ & $101.58 \pm 34.65$ \\
\hline$t$ & & 10.69 & 9.87 & 11.48 & 9.53 \\
\hline$P$ & & $<0.01$ & $<0.01$ & $<0.01$ & $<0.01$ \\
\hline
\end{tabular}

Comparison of serum endostatin and FGF-19 levels in the chemotherapeutic sensitivity and chemotherapeutic resistance groups after chemotherapy

Before and after chemotherapy, the levels of serum endostatin and FGF-19 in the chemotherapeutic sensitivity group were significantly lower than those in the chemotherapeutic resistance group, $(\mathrm{P}<0.05$ and $\mathrm{P}<0.01$, respectively; Table 2$)$.

Table 2. Comparison of the levels of serum endostatin and FGF-19 in two groups after chemotherapy.

\begin{tabular}{|c|c|c|c|c|c|}
\hline \multirow[t]{2}{*}{ Groups } & \multirow[t]{2}{*}{ Cases } & \multicolumn{2}{|c|}{ Endostatin $(\mu \mathrm{g} / \mathrm{L})$} & \multicolumn{2}{|c|}{ FGF-19 (ng/L) } \\
\hline & & Before chemotherapy & After chemotherapy & Before chemotherapy & After chemotherapy \\
\hline Sensitivity group & 21 & $23.18 \pm 9.64$ & $14.61 \pm 4.42$ & $210.74 \pm 36.48$ & $120.64 \pm 24.12$ \\
\hline Resistance group & 39 & $31.15 \pm 7.14$ & $20.13 \pm 5.71$ & $281.14 \pm 41.63$ & $158.14 \pm 25.51$ \\
\hline$t$ & & 8.25 & 7.48 & 8.06 & 8.14 \\
\hline $\mathrm{P}$ & & $<0.01$ & $<0.01$ & $<0.01$ & $<0.01$ \\
\hline
\end{tabular}

\section{Predictive value of serum endostatin and FGF-19 levels for the chemotherapeutic} sensitivity of acute myeloid leukemia patients

ROC curve analysis of serum endostatin and FGF-19 levels before chemotherapy showed that the sensitivity predicting chemotherapeutic sensitivity was $92.16 \%$ and the speci- 
ficity was $65.43 \%$ when endostatin reached $30.285 \mu \mathrm{g} / \mathrm{L}$ (Figure 1). When FGF-19 reached $276.140 \mathrm{ng} / \mathrm{L}$, the sensitivity predicting chemotherapeutic sensitivity was $94.43 \%$ and the specificity was $69.38 \%$. There was no significant difference between these two results (Table 3 ).

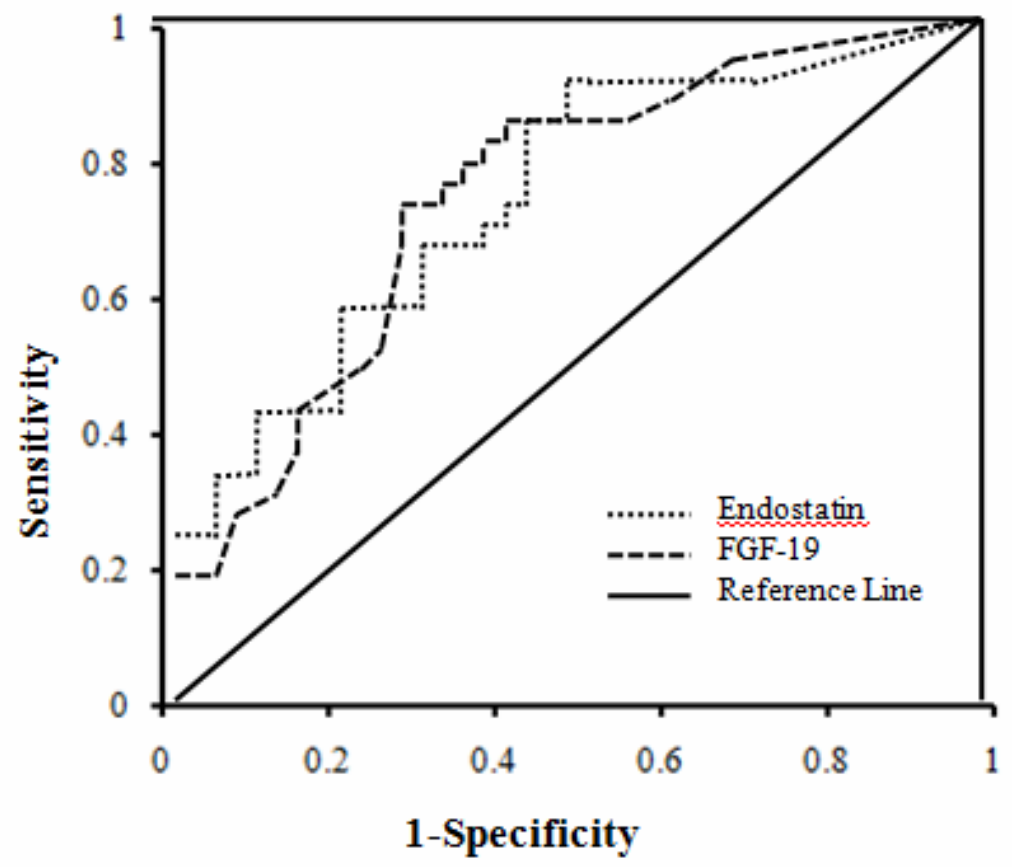

Figure 1. ROC curve of predictive value of the levels of serum endostatin and FGF-19 on chemotherapeutic sensitivity before chemotherapy.

Table 3. Predictive value of the levels of serum endostatin and FGF-19 on chemotherapeutic sensitivity of the patients with acute myeloid leukemia.

\begin{tabular}{lcccc}
\hline Indexes & Critical value & Area under curve & Sensibility (\%) & Specificity (\%) \\
\hline Endostatin & $30.285 \mu \mathrm{g} / \mathrm{L}$ & 0.701 & 92.16 & 65.43 \\
FGF-19 & $276.140 \mathrm{ng} / \mathrm{L}$ & 0.712 & 94.43 & 69.38 \\
\hline
\end{tabular}

\section{DISCUSSION}

Acute myeloid leukemia is a heterogeneous malignant disease of the blood and includes acute leukemia originating from non-lymphocytes (Trifilio et al., 2013). Although hematopoietic stem cell transplantation can help acute myeloid leukemia patients achieve long-term disease-free survival or even a cure, it is an expensive treatment, and hence, is not desired by all patients. Chemotherapy is still the main treatment for acute myeloid leukemia (Wu et al., 2012; Champlin, 2013; Martner et al., 2013). There is continuous in-depth research into the cytogenetics of this disease, its mechanism of occurrence and development, and the effectiveness of chemotherapeutic drugs. Consequently, the remission and overall survival 
rates after chemotherapy are increasing for these patients; some patients with acute myeloid leukemia can obtain long-term disease-free survival (Penack, 2013; Cagnetta et al., 2014). In addition, the prognosis of some patients was good, but did not correspond with the clinical response to therapy; also, the treatment effect was poor, the symptoms were difficult to relieve, and symptoms recurred easily (Chromik et al., 2014). Therefore, it is important to identify effective methods to evaluate curative effects and prognosis after chemotherapy. Endostatin was discovered in 1997, and was considered one of the most active angiogenesis inhibitors. It can effectively inhibit the growth of endothelial cells, and has been associated with the development and prognosis of malignant tumor diseases (Fung et al., 2013; Watts et al., 2014). FGF-19 belongs to an endocrine subfamily, and it is expressed in the terminal ileum, brain, cartilage, kidney, and other organs; it is important for the metabolism of nutrients. Studies showed that its expression was altered in leukemia patients (Cojoc et al., 2014; Haley and Kim, 2014). Endostatin is currently considered one of the most effective endogenous vascular growth inhibitors. Research has shown that endostatin was associated with the onset and progression of leukemia (Mittal et al., 2014). Changes in serum endostatin and FGF-19 in patients with acute myeloid leukemia may affect chemotherapeutic sensitivity and prognosis; however, few studies have examined this. It is important to clarify any changes in serum endostatin and FGF19 levels in these patients, and to determine their effects on remission rates and survival time.

The results of this study showed that the levels of serum endostatin and FGF-19 in acute myeloid leukemia patients were increased compared with the normal population. The levels of serum endostatin and FGF-19 in the chemotherapeutic sensitivity group were significantly lower than that in the chemotherapeutic resistance group, which suggested that chemotherapy based on cytarabine over a certain time period can reduce these levels in patients with acute myeloid leukemia. Therefore, the levels of serum endostatin and FGF-19 may be associated with the pathogenesis of acute myeloid leukemia and treatment effects. ROC curve analysis showed that when endostatin reached $30.285 \mu \mathrm{g} / \mathrm{L}$, the sensitivity predicting chemotherapeutic sensitivity was $92.16 \%$ and the specificity was $65.43 \%$, and when FGF-19 reached $276.140 \mathrm{ng} / \mathrm{L}$, the sensitivity was $94.43 \%$ and the specificity was $69.38 \%$. There was no significant difference between the two results, which confirmed that the levels of serum endostatin and FGF-19 in patients with acute myeloid leukemia could be used as predictors of chemotherapeutic sensitivity. These levels may be associated with treatment effects and the prognosis of the patients with acute myeloid leukemia, and this supports the hypothesis that serum endostatin and FGF-19 in patients with acute myeloid leukemia may affect chemotherapeutic sensitivity and prognosis. For patients with higher levels of serum endostatin and FGF-19, options include comprehensive treatment or hematopoietic stem cell transplantation depending on their clinical characteristics. Preoperative analysis of serum endostatin and FGF-19 levels may help predict the chemotherapeutic effect and the selection of a treatment plan for acute myeloid leukemia patients. In this study there were small sample sizes, short chemotherapy duration and observation time. Clarifying the effects of changes in serum endostatin and FGF-19 levels on chemotherapeutic sensitivity in acute myeloid leukemia patients will require long-term observation and in-depth research with a larger sample size.

In conclusion, the levels of serum endostatin and FGF-19 were higher in patients with acute myeloid leukemia. Serum endostatin and FGF-19 in patients with acute myeloid leukemia can be used as predictors of chemotherapeutic sensitivity, and may be important for determining the prognosis. 


\section{REFERENCES}

Bernal T, Diez-Campelo M, Godoy V, Rojas S, et al. (2014). Role of minimal residual disease and chimerism after reduced-intensity and myeloablative allo-transplantation in acute myeloid leukemia and high-risk myelodysplastic syndrome. Leuk. Res. 38: 551-556.

Cagnetta A, Adamia S, Acharya C, Patrone F, et al. (2014). Role of genotype-based approach in the clinical management of adult acute myeloid leukemia with normal cytogenetics. Leuk. Res. 38: 649-659.

Champlin R (2013). Reduced intensity allogeneic hematopoietic transplantation is an established standard of care for treatment of older patients with acute myeloid leukemia. Best Pract. Res. Clin. Haematol. 26: 297-300.

Chromik J, Safferthal C, Serve H and Fulda S (2014). Smac mimetic primes apoptosis-resistant acute myeloid leukaemia cells for cytarabine-induced cell death by triggering necroptosis. Cancer Lett. 344: 101-109.

Cojoc M, Mäbert K, Muders MH and Dubrovska A (2014). A role for cancer stem cells in therapy resistance: Cellular and molecular mechanisms. Semin. Cancer Biol. 31: 16-27.

Fung FY, Li M, Breunis H, Timilshina N, et al. (2013). Correlation between cytokine levels and changes in fatigue and quality of life in patients with acute myeloid leukemia. Leuk. Res. 37: 274-279.

Gacche RN and Meshram RJ (2014). Angiogenic factors as potential drug target: efficacy and limitations of anti-angiogenic therapy. Biochim. Biophys. Acta 1846: 161-179.

Haley EM and Kim Y (2014). The role of basic fibroblast growth factor in glioblastoma multiforme and glioblastoma stem cells and in their in vitro culture. Cancer Lett. 346: 1-5.

Lin PH, Lin CC, Yang HI, Li LY, et al. (2013). Prognostic impact of allogeneic hematopoietic stem cell transplantation for acute myeloid leukemia patients with internal tandem duplication of FLT3. Leuk. Res. 37: 287-292.

Liu XY, Li HG, Zhang KJ and Gu JF (2012). Strategy of Cancer Targeting Gene-Viro-Therapy (CTGVT) a trend in both cancer gene therapy and cancer virotherapy. Curr. Pharm. Biotechnol. 13: 1761-1767.

Martner A, Thorén FB, Aurelius J and Hellstrand K (2013). Immunotherapeutic strategies for relapse control in acute myeloid leukemia. Blood Rev. 27: 209-216.

Mittal K, Ebos J and Rini B (2014). Angiogenesis and the tumor microenvironment: vascular endothelial growth factor and beyond. Semin. Oncol. 41: 235-251.

Moiseev IS, Lapin SV, Surkova EA, Lerner MY, et al. (2013). Level of vascular endothelial growth factor predicts both relapse and nonrelapse mortality after allogeneic hematopoietic stem cell transplantation. Biol. Blood Marrow Transplant. 19: 1677-1682.

Oberoi S, Lehrnbecher T, Phillips B, Hitzler J, et al. (2014). Leukapheresis and low-dose chemotherapy do not reduce early mortality in acute myeloid leukemia hyperleukocytosis: a systematic review and meta-analysis. Leuk. Res. 38: 460-468.

Penack O (2013). CH 20: The endothelium in graft-versus-host disease and graft-versus-leukemia. In: Immune Biology of Allogeneic Hematopoietic Stem Cell Transplantation (Socie G and Blazar BR, eds.). Academic Press, 479-492.

Rotschafer JH, Hu S, Little M, Erickson M, et al. (2013). Modulation of neural stem/progenitor cell proliferation during experimental Herpes Simplex encephalitis is mediated by differential FGF-2 expression in the adult brain. Neurobiol. Dis. 58: 144-155.

Trifilio S, Zhou Z, Altman J, Frankfurt O, et al. (2013). Dose-intense etoposide-cyclophosphamide without stem cell transplantation for patients with intermediate and high cytogenetic risk primary refractory and relapsed acute myeloid leukemia. Leuk. Res. 37: 872-876.

Watts JM, Wang XV, Litzow MR, Luger SM, et al. (2014). Younger adults with acute myeloid leukemia in remission for $\geq 3$ years have a high likelihood of cure: The ECOG experience in over 1200 patients. Leuk. Res. 38: 901-906.

Won D, Chi HS, Shim H, Jang S, et al. (2013). The prognostic impact of c-KIT mutation in systemic mastocytosis associated with acute myeloid leukaemia patients. Leuk. Res. 37: 883-888.

Wu SD, Ma YS, Fang Y, Liu LL, et al. (2012). Role of the microenvironment in hepatocellular carcinoma development and progression. Cancer Treat. Rev. 38: 218-225.

Xia Y, Lu RN and Li J (2012). Angiogenic factors in chronic lymphocytic leukemia. Leuk. Res. 36: 1211-1217. 